\title{
Multiscale modelling of hardening in BCC crystal plasticity
}

\author{
L. Stainier, A.M. Cuitino ${ }^{1}$ and M. Ortiz ${ }^{2}$
}

\author{
Département Aérospatiale, Mécanique et Matériaux, Université de Liège, LTAS-MCT, \\ Chemin des Chevreuils 1, 4000 Liège, Belgium \\ ${ }^{1}$ Department of Mechanical and Aerospace Engineering, Rutgers University, 98 Brett Roads, \\ Piscataway, NJ 08854, U.S.A. \\ ${ }^{2}$ Graduate Aeronautical Laboratories, California Institute of Technology, \\ 1200 E. California BIVd., MS 105-50, Pasadena, CA 91125, U.S.A.
}

\begin{abstract}
The mechanical behavior of polycrystalline metals can be successfully modeled by macroscopic theories, such as Von Mises plasticity. On the other hand, numerous studies can be performed on the atomic scale, either by atomistic or dislocation dynamics models. The proposed model attempts to bridge those two scales by deriving constitutive relations between slip strains, dislocation densities and resolved shear stresses on crystallographic planes, from mechanisms of deformation playing at the level of the dislocation line. The resulting "mesoscopic" hardening relations are controlled by dislocation self energies and junctions strengths. Temperature and strain rate dependence result from the presence of thermally activated mechanisms such as Peieris barriers or pair annihilation by cross slip. A set of material parameters is identified for Tantalum by fitting the numerical stress strain curves from these tests with experimental results gathered in the literature. These parameters prove to be in very good agreement with the values which can be derived from molecular dynamics computations.
\end{abstract}

\section{INTRODUCTION}

The present paper is concerned with the development of a micromechanical model of the hardening, ratesensitivity and thermal softening of b.c.c. crystals. We place primary emphasis on the derivation of closedform analytical expressions describing the macroscopic behavior of the crystals amenable to implementation as constitutive relations within a standard finite-element code. In developing the model, we follow the well-established paradigm of micromechanical modeling, consisting of: the identification of the dominant or rate-limiting 'unit' processes operating at the microscale; the identification of the macroscopic forces driving the unit processes; the analysis of the response of the unit processes to the macroscopic driving forces; and the determination of the average or macrocospic effect of the combined operation of all the micromechanical unit processes. We show in this article that the meticulous application of this paradigm renders truly predictive models of the mechanical behavior of complex systems. In particular we predict the hardening of Ta single crystal and its dependency for a wide range of temperatures and strain rates. The feat of this approach is that predictions from these atomistically informed models recover most of the macroscopic characteristic features of the available experimental data, without a priori knowledge of such experimental tests. This approach then provides a procedure to forecast the mechanical behavior of material in extreme conditions where experimental data is simply not available or very difficult to collect.

In formulating the present model we specifically consider the following unit processes: double-kink formation and thermally activated motion of kinks; the close-range interactions between primary and forest dislocations, and the subsequent formation of jogs; the percolation motion of dislocations through a random 


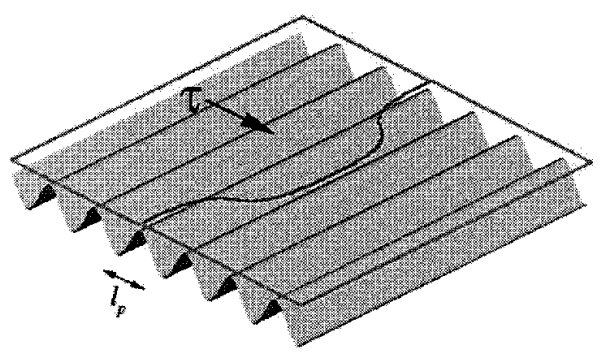

(a)

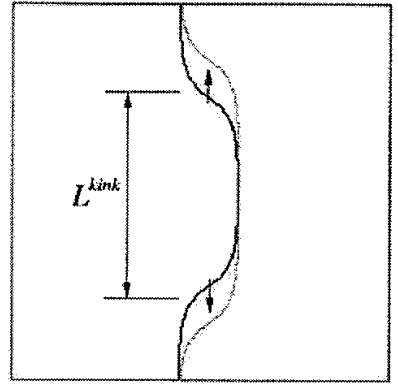

(b)

Figure 1: Schematic of the double-kink mechanism.

array of forest dislocations introducing short-range obstacles of different strengths; dislocation multiplication due to breeding by double cross-slip; and dislocation pair annihilation. We believe that this forms an 'irreducible' set of unit processes, in that each of these processes accounts for-and is needed for matchingsalient and clearly recognizable features of the experimental record. We validate the micromechanical model by recourse to detailed comparisons with the uniaxial tension tests on Ta single crystals of Mitchell and Spitzig [1]. The model is found to capture salient features of the behavior of Ta crystals.

\section{UNIT MECHANISMS}

In this section, we introduce the set of controlling unit processes which have been identified for describing the mechanical response of high-purity b.c.c. single crystals, in particular for Tantalum. A detailed description of the model, including comparison with experimental data is given in [2].

\subsection{Dislocation Mobility: Double-Kink Formation and Thermally Activated Motion of Kinks}

In b.c.c. crystals, the core of screw dislocation segments relaxes into low-energy non-planar configurations. This introduces deep valleys into the Peierls energy function aligned with the Burgers vector directions and possessing the periodicity of the lattice. At low temperatures, the dislocations tend to adopt lowenergy configurations and, consequently, the dislocation population predominantly consists of long screw segments. In order to move a screw segment normal to itself, the dislocation core must first be constricted, which requires a substantial supply of energy. Thus, the energy barrier for the motion of screw segments, and the attendant Peierls stress, may be expected to be large, and the energy barrier for the motion of edge segments to be comparatively smaller. This suggests that the rate-limiting mechanism for dislocation motion is the thermally activated motion of kinks along screw segments $([3,4,5])$.

At sufficiently high temperatures and under the application of a resolved shear stress $\tau>0$, a doublekink may be nucleated with the assistance of thermal activation (e. g., $[6,7,8]$ ), and the subsequent motion of the kinks causes the screw segment to effectively move forward, Fig. 1. Under this conditions the following expression for the effective temperature and strain-rate dependent Peierls $\tau_{p}$ is obtained:

$$
\tau_{P}=\frac{\tau_{0}}{\beta E^{\text {kink }}} \operatorname{asinh}\left(\frac{\dot{\gamma}}{\dot{\gamma}_{0}^{\text {kink }}} \mathrm{e}^{\beta \mathrm{E}^{\text {kink }}}\right)
$$

where the effective Peierls stress is given by

$$
\tau_{0}=\frac{E^{\text {kink }}}{b L^{\text {kink }} l_{P}}
$$




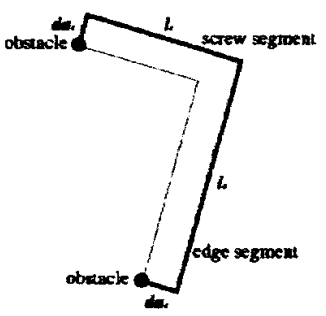

Figure 2: Bow-out mechanism for a dislocation segment bypassing an obstacle pair

and the reference strain is defined as

$$
\dot{\gamma}_{0}^{\text {kink }}=2 b \rho l_{P} \nu_{D}
$$

In the preceeding equations, $b$ is the Burgers vector, $\rho$ is the dislocation density, $\beta=1 / k_{B} T, k_{B}$ is Boltzmann's constant, $T$ is the absolute temperature, and $\nu_{D}$ is the attempt frequency which may be identified with the Debye frequency to a first approximation. Also, $l_{P}$ is the distance between two consecutive Peierls valleys. For b.c.c. crystals, $l_{P}=\sqrt{2 / 3} a$ if the slip plane is $\{110\}, l_{P}=\sqrt{2} a$, if the slip plane is $\{112\}$, and $l_{P}=\sqrt{8 / 3} a$ if the slip plane is $\{123\}$, where $a$ is the cubic lattice size [9]. Finally, $E^{\text {kink }}$ is the energy of formation of a kink-pair and $L^{\text {kink }}$ is the length of an incipient double kink. The formation energy $E^{\text {kink }}$ and the length $L^{\text {kink }}$, which cannot be reliably estimated from elasticity since the energy is composed mostly of core region, can, however, be accurately computed by recourse to atomistic models. Modeling of this first unit process renders the first 2 material properties amenable of atomistic calculations.

\subsection{Dislocation Interactions: Obctacle-Pair Strength and Obstacle Strength}

\subsubsection{Obstacle-Pair Strength}

We begin by treating the case of infinitely strong obstacles. In this case, pairs of obstacles pin down dislocation segments, which require a certain threshold resolved shear stress $s$ in order to overcome the obstacle pair. The lowest-energy configuration of unstressed dislocation segments spanning an obstacle pair is a step of the form shown as the thin line in Fig. 2. Under these conditions, the bow-out mechanism by which a dislocation segment bypasses an obstacle pair may be expected to result in the configuration shown in Fig. 2 (bold line). If the edge-segment length is $l_{e}$, a displacement $d a_{e}$ of the dislocation requires a supply of energy equal to $2 U^{\text {screw }} d a_{e}+b \tau_{P}^{\text {edge }} l_{e} d a_{e}$ in order to overcome the Peierls resistance $\tau_{P}^{\text {edge }}$ and to extend the screw segments. The corresponding energy release is $b \tau l_{e} d a_{e}$. Similar contributions result from a displacement $d a_{s}$ of the screw-segment of length $l_{s}$. Retaining dominant terms the obstacle-pair strength is

$$
s=\tau_{P}^{\text {screw }}+\frac{2 U^{\text {edge }}}{b l_{s}}
$$

The obstacle-pair strength can be therefore estimated by quantifying $\tau_{P}, l_{s}$ and $U^{\text {edge }}$. An expression for the Peierls stress $\tau_{P}$ is given in Eq. (1), the distance between obstacles along the screw direction $l_{s}$ is estimated by statistics assuming a random obstacle distribution and the core energy per unit length in the edge direction $U^{\text {edge }}$ can be obtained by atomistic calculations.

\subsubsection{Obstacle Strength}

For purposes of the present theory, we specifically concern ourselves with short-range interactions between dislocations which can be idealized as point defects. For simplicity, we consider the case in which each 


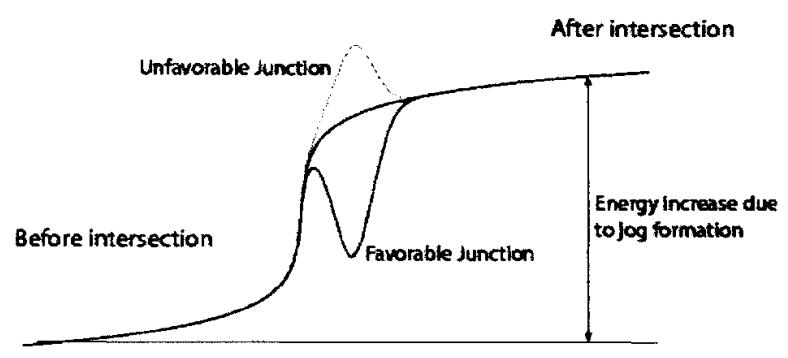

Figure 3: Schematic of energy variation as a function of a reaction coordinate during dislocation intersection and crossing.

intersecting dislocation acquires a jog. The energy of a pair of crossing dislocations is schematically shown in Fig. 3 as a function of some convenient reaction coordinate, such as the distance between the dislocations. The interaction may be repulsive, resulting in an energy barrier, or attractive, resulting in a binding energy, Fig. 3. In the spirit of an equilibrium theory, here we consider only the final reaction product, corresponding to a pair of jogged dislocations at infinite distance from each other, and neglect the intermediate states along the reaction path. In addition, we deduce the strength of the obstacles directly from the energy supply required to attain the final state, i. e. the jog-formation energy. Based on energy and mobility considerations already discussed, we may expect the preponderance of forest dislocations to be of screw character, and the mobile dislocation segments to be predominantly of edge character. We therefore restrict our analysis to intersections between screw and edge segments. The geometry of the crossing process is schematically shown in Fig. 4(a). Each dislocation acquires a jog equal to the Burgers vector of the remaining dislocation. The energy expended in the formation of the jogs may be estimated as

$$
E_{\alpha \beta}^{j o g s} \sim \begin{cases}b U^{\text {screw }}\left[1-r \cos \theta^{\alpha \beta}\right] & \text { if } \mathbf{b}^{\alpha}=\mathbf{b}^{\beta} \\ b U^{\text {screw }}\left[2 r-\cos \left(\theta^{\beta \alpha}\right)-r \cos \theta^{\alpha \beta}\right] & \text { otherwise }\end{cases}
$$

where $r=U^{\text {edge }} / U^{\text {screw }}$ is the ratio of screw to edge dislocation line energies. This ratio, when computed by atomistic calculations [10], renders a value of $r=1.77$ for Ta. A derivation entirely analogous to that leading to Eq. (1) yields the following expression for the strength of an obstacle in the slip system $\alpha$ produced by a forest segment in the system $\beta$

$$
s^{\alpha \beta}=\frac{s_{0}^{\alpha \beta}}{\beta E_{\alpha \beta}^{\mathrm{jog}}} \operatorname{asinh}\left(\frac{\dot{\gamma}^{\alpha}}{\dot{\gamma}_{0}^{\alpha}} \mathrm{e}^{\beta \mathrm{E}_{\alpha \beta}^{\mathrm{jog}}}\right)
$$

where the strength at zero temperature and the reference strain rate are given by

$$
s_{0}^{\alpha \beta}=\frac{E_{\alpha \beta}^{\mathrm{jog}}}{b \bar{l}^{\alpha} L^{\mathrm{junct}}} \quad \text { and } \quad \dot{\gamma}_{0}^{\alpha}=2 \rho^{\alpha} b \bar{l}^{\alpha} \nu_{D}
$$

The lengths $\bar{l}^{\alpha}$ and $L^{\text {junct }}$ describe the geometry of the junction as illustrated in Fig. 4(b). These values, which have been estimated to be of the order of few $b$ in the present case, can also be obtained by atomistic models.

\subsection{Dislocation Evolution: Multiplication and Attrition}

Processes resulting in changes in dislocation density include production by fixed sources, such as FrankRead sources, breeding by double cross slip and pair annihilation (see [11] for a review). Although the 


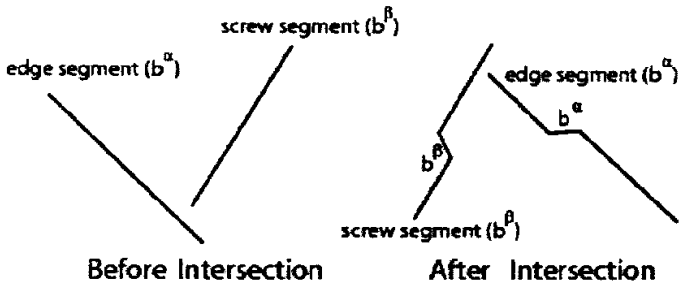

(a) formation of jogs

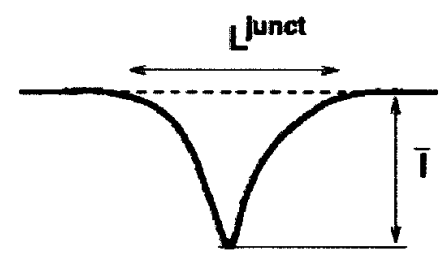

(b) dislocation line overcoming a junction

Figure 4: Schematic of jog formation during dislocation intersection

operation of fixed Frank-Read sources is quickly eclipsed by production due to cross slip at finite temperatures, it is an important mechanisms at low temperatures. The double cross-slip, fixed Frank-Read sources and pair annihilation mechanisms are next considered in turn.

\subsubsection{Dislocation Multiplication: Füed Frank-Reed and Breeding by Cross Glide}

The rate of dislocation multiplication in a given slip system $\alpha$ produced by fixed Frank-Reed sources and by breeding by cross glide is written as

$$
b \dot{\rho}^{\alpha}=\lambda_{0} \sqrt{\rho^{\alpha}} \dot{\gamma}^{\alpha}
$$

where $\lambda_{0}$ is a constant associated with the fixed Frank-Read production; this parameter is rather topological than material dependent.

\subsubsection{Attrition: Pair Annihilation}

The rate of dislocation attrition due to pair annihilation may finally be estimated as:

$$
b \dot{\rho}^{\alpha}=-\kappa \rho^{\alpha} \dot{\gamma}^{\alpha}
$$

where $\kappa$ is the effective annihilation distance. This is the maximum distance at which two screw segments with opposite direction and forced to move with a velocity $v=\dot{\gamma} / b \rho$ will annihilate. This distance can be estimated by simply equating the time required for trapping and escaping. Trapping is governed by the elastic interaction forces (attraction) while escaping by the applied strain rate. Then,

$$
\frac{1}{\kappa}=\frac{1}{\kappa_{c}}+\frac{1}{\kappa_{0}\left(A+\sqrt{A^{2}+1}\right)}
$$

where

$$
A=\mathrm{e}^{-\beta E^{\mathrm{jog}}} \beta E^{\mathrm{jog}} \dot{\gamma}_{0}^{\mathrm{jog}} / \dot{\gamma}^{\alpha} \quad \text { and } \quad \dot{\gamma}_{0}^{\mathrm{jog}}=2 b \rho l_{P} \nu_{D}
$$

are respectively a factor depending on the strain rate and temperature and a reference slip-strain rate, while $\kappa_{c}$ is the cut-off value corresponding the effective screening distance. It follows that the critical pairannihilation distance $\kappa$ decreases with increasing strain rate and decreasing temperature. The rate of annihilation is then modulated by the nucleation energy of a jog $E^{\mathrm{jog}}$ which can be calculated from atomistic simulations. 
Table 1: Material parameters for Tantalum

\begin{tabular}{|c|c|c|c|}
\hline Parameter & Fitted set & Atomistic set & Units \\
\hline$E^{\text {kink }}$ & 0.70 & 0.725 & {$[\mathrm{eV}]$} \\
\hline$L^{\mathrm{kink}} / b$ & 13 & 17 & \\
\hline$U^{\text {edge }} / \mu b^{2}\left(^{*}\right)$ & 0.2 & 0.216 & \\
\hline$U^{\text {edge }} / U^{\text {screw }}$ & 1.77 & 1.77 & \\
\hline $\bar{l} / b$ & 5 & 5 & \\
\hline$L^{\text {junct }} / b$ & 20 & 20 & \\
\hline$E^{\text {cross }}$ & 0.67 & 0.725 & {$[\mathrm{eV}]$} \\
\hline$L^{\text {cross }} / b$ & 13 & 17 & \\
\hline$\lambda_{\text {FR }}$ & 2.3 & $4.5^{* *}$ & \\
\hline$\kappa_{c} / b$ & 1250 & $500^{* *}$ & \\
\hline
\end{tabular}

\section{COMPARISON WITH EXPERIMENT}

We proceed to validate the theory against the uniaxial tests on Ta single crystals of Mitchell and Spitzig [1]. In these tests, 99.97\%-pure Ta specimens were loaded in tension along the [213] crystallographic axis, at various combinations of temperature and strain rate. In particular we considered temperatures ranging from $296 \mathrm{~K}$ to $573 \mathrm{~K}$, and strain rates ranging from $10^{-1} \mathrm{~s}^{-1}$ to $10^{-5} \mathrm{~s}^{-1}$. The numerical procedure employed for the integration of the constitutive equations has been described elsewhere [12]. The constitutive update is fully implicit, with the active systems determined iteratively so as to minimize an incremental work function. All stress-strain curves are reported in terms of nominal stress and engineering strain. Two different sets of material properties were used for the numerical simulations. The first set was obtained by fitting the simulation results to the experimental results, using a simple trial and error procedure. Table 1 identifies the subset of parameters which are also amenable to direct calculation by atomistic based methods. The table lists the parameter values obtained by these methods, as described in [13], in parallel with the yalues obtained by the fitting approach. Thus, in the second set of properties which was used for numerical simulations, atomistic-based values replace fit-based values, when available. This is the case for the edge and screw dislocation self-energies, as well as the kink-pair formation energy and length. Clearly, those two sets don't differ by much, which strongly support the validity of the advertised multiscale paradigm. For the two parameters linked to dislocation density evolution, atomistic simulations were not yet available at time of writing, and the values listed here were obtained by fitting. For a complete list of parameters for the model, the reader should refer to [2].

Figs. 5 show the predicted and measured stress-strain curves for a [213] Ta crystal over a range of temperatures and strain rates. One can compare, from top to bottom: the experimental results, the results obtained after fitting the parameters, and the results obtained with atomistic-based parameters. It is evident from these figures that the model, with both sets of parameters, captures salient features of the behavior of Ta crystals such as: the dependence of the initial yield point on temperature and strain rate; the presence of a marked stage I of easy glide, specially at low temperature and high strain rates; the sharp onset of stage II hardening and its tendency to shift towards lower strains, and eventually disappear, as the temperature increases or the strain rate increases; the parabolic stage II hardening at low strain rates or high temperatures; the stage II softening at high strain rates or low temperatures; the trend towards saturation at high strains; and the temperature and strain-rate dependence of the saturation stress. Thus, the predictive approach based on atomistic methods clearly shows its capacity to produce results matching the experimental evidence. 

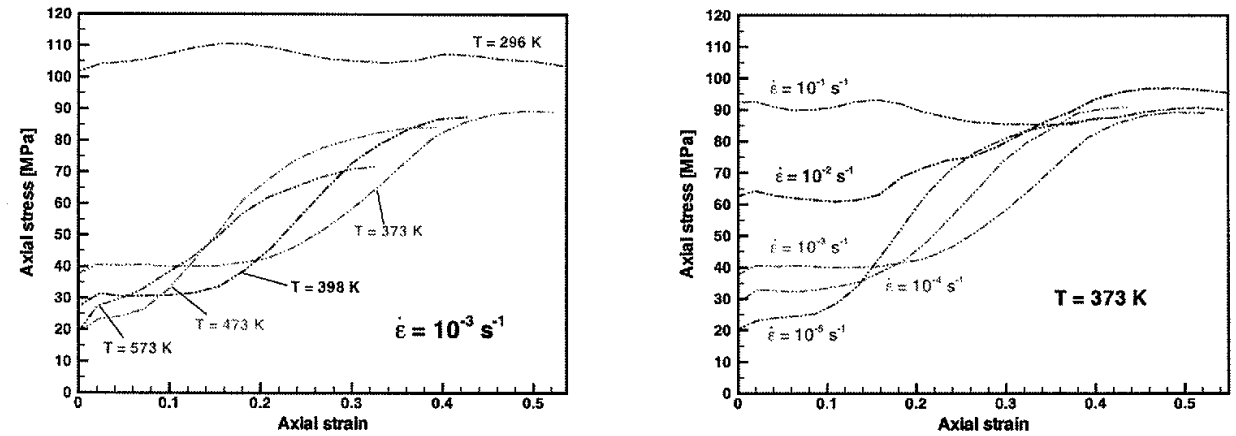

(a) Experimental data of Mitchell \& Spitzig [1]
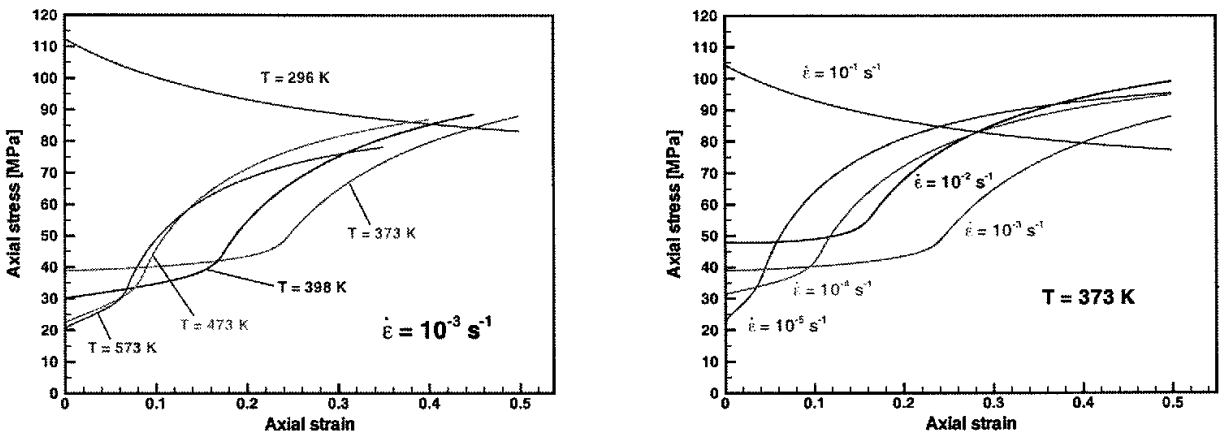

(b) Predictions of the model with fitted parameters
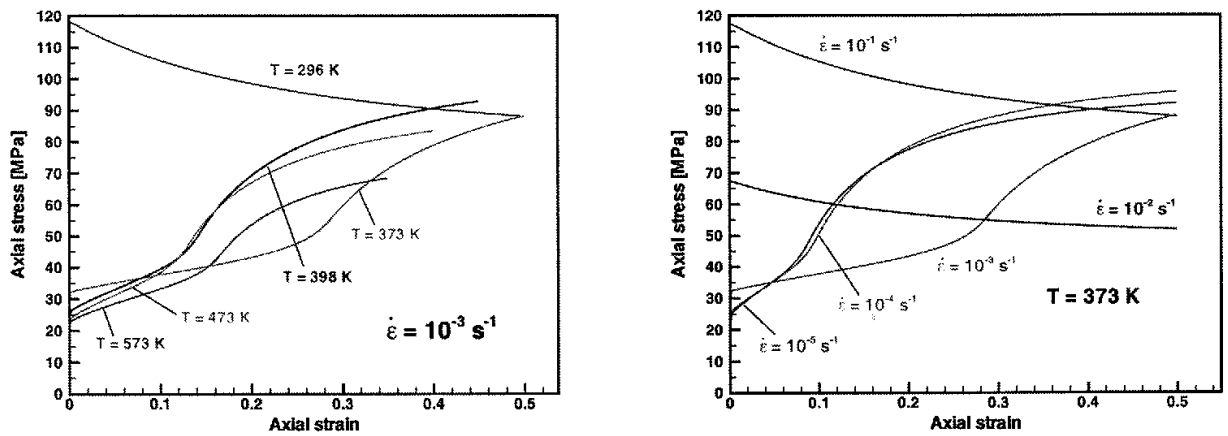

(c) Predictions of the model with atomistic parameters

Figure 5: Temperature and strain-rate dependence of stress-strain curves for [213] Ta single crystal. 


\section{SUMMARY AND CONCLUSIONS}

We have developed a micromechanical model of the hardening, rate-sensitivity and thermal softening of b.c.c. crystals. The model is predicated upon the consideration of an 'irreducible' set of unit processes, consisting of: double-kink formation and thermally activated motion of kinks; the close-range interactions between primary and forest dislocation, leading to the formation of jogs; the percolation motion of dislocations through a random array of forest dislocations introducing short-range obstacles of different strengths; dislocation multiplication due to breeding by double cross-slip; and dislocation pair-annihilation. Each of these processes accounts for-and is needed for matching-salient and clearly recognizable features of the experimental record. In particular, on the basis of detailed comparisons with the experimental data of Mitchell and Spitzig [1], the model is found to capture salient features of of the behavior of Ta crystals.

\section{Acknowledgements}

The support of the DOE through Caltech's ASCI Center for the Simulation of the Dynamic Response of Materials is gratefully acknowledged. LS also wishes to acknowledge support from the Belgian National Fund for Scientific Research (FNRS).

\section{REFERENCES}

[1] T.E. Mitchell and W.A. Spitzig. Three-stage hardening in tantalum single crystals. Acta Metallurgica, 13:1169-1179, 1965.

[2] A. M Cuitiño L. Stainer and M. Ortiz. Micromechanical modeling of hardening, rate sensitivity and thermal softening in bcc single crystals. Journal of the Mechanics and Physics of Solids, 50:1511$1545,2002$.

[3] P. B. Hirsch. In 5th International Conference on Crystallography, page 139. Cambridge University, 1960.

[4] A. Seeger and P. Schiller. Acta Metallurgica, 10:348, 1962.

[5] J. P. Hirth and J. Lothe. Theory of Dislocations. McGraw-Hill, New York, 1968.

[6] J.P. Hirth and R.G. Hoagland. Nonlinearities in the static energetics and in the kinematics of dislocations. Physica D, 66(1-2):71-77, 1993.

[7] W. Xu and J.A. Moriarty. Accurate atomistic simulations of the peierls barrier and kink-pair formation energy for < $111>$ screw dislocations in bcc Mo. Computational Materials Science, 9(3-4):348-356, 1998.

[8] J.A. Moriarty, W. Xu, P. Soderlind, J. Belak, L.H. Yang, and J. Zhu. Atomistic simulations for multiscale modeling in bcc metals. Journal of Engineering Materials and Technology-Transactions of the ASME, 121(2):120-125, 1999.

[9] A. Seeger and L. Hollang. The flow-stress asymmetry of ultra-pure molybdenum single crystals. Materials Transactions JIM, 41(1):141-151, 2000.

[10] Guofeng Wang, Alejandro Strachan, Tahir Cagin and William A. Goddard III. Kinks in a $<111>$ screw dislocation in Ta. Journal of Computer Aided Materials Design, 8(2-3):117-125, 2001.

[11] D. Kuhlmann-Wilsdorf. Theory of plastic deformation: properties of low energy dislocation structures. Materials Science and Engineering, A113:1, 1989.

[12] M. Ortiz and L. Stainier. The variational formulation of viscoplastic constitutive updates. Computer Methods in Applied Mechanics and Engineering, 171(3-4):419-444, 1999.

[13] A. Cuitiño, L. Stainier, G. Wang, A. Strachan, T. Cagin, W.A. Goddard III and M. Ortiz. A multiscale approach for modelling crystalline solids. J. Computer-Aided Materials Design, 8(2-3):127-149, 2001. 